\title{
Level of mortality risk for babies born preterm or with a small weight for gestation in a tertiary hospital of Nepal
}

\author{
Ashish $\mathrm{KC}^{1,2^{*}}$, Johan Wrammert ${ }^{1}$, Viktoria Nelin ${ }^{1}$, Uwe Ewald ${ }^{1}$, Robert Clark ${ }^{3}$ and Mats Målqvist ${ }^{1}$
}

\begin{abstract}
Background: Globally, 15 million babies were born prematurely in 2012, with $37.6 \%$ of them in South Asia. About 32.4 million infants were born small for gestational age (SGA) in 2010, with more than half of these births occurring in South Asia. In Nepal, 14 \% of babies were born preterm and $39.3 \%$ were born SGA in 2010. We conducted a study in a tertiary hospital of Nepal to assess the level of risk for neonatal mortality among babies who were born prematurely and/or SGA.

Methods: This case-control study was completed over a 15-month period between July 2012 and September 2013. All neonatal deaths that occurred during the study period were included as cases and $20 \%$ of women with live births were randomly selected as referents. Information on potential risk factors was taken from medical records and interviews with the women. Logistic regression analyses were conducted to determine the level of risk for neonatal mortality among babies born preterm and/or SGA.

Results: During this period, the hospital had an incidence of preterm birth and SGA of 8.1 and $37.5 \%$, respectively. In the multivariate model, there was a 12-fold increased risk of neonatal death among preterm infants compared to term. Babies who were SGA had a $40 \%$ higher risk of neonatal death compared to those who were not. Additionally, babies who were both preterm and SGA were 16 times more likely to die during the neonatal period.

Conclusions: Our study showed that the risk of neonatal mortality was highest when the baby was born both preterm and SGA, followed by babies who were born preterm, and then by babies who were SGA in a tertiary hospital in Nepal. In tertiary care settings, the risk of mortality for babies who are born preterm and/or SGA can be reduced with low-cost interventions such as Kangaroo Mother Care or improved management of complications through special newborn care or neonatal intensive care units. The risk of death for babies who are born prematurely and/or SGA can thus be used as an indicator to monitor the quality of care for these babies in health facility settings.
\end{abstract}

Clinical trial registration: ISRCTN97846009

\section{Background}

In 2012, 2.9 million neonatal deaths (deaths in the first 28 days after birth) occurred globally; of these, almost $34 \%$ ( 1 million) were directly caused by complications due to preterm birth (before 37 gestational weeks) [1]. Additionally, preterm birth was a risk factor for approximately

\footnotetext{
* Correspondence: aaashis7@yahoo.com

'International Maternal and Child Health, Department of Women's and

Children's Health, Uppsala University, Uppsala, Sweden

2United Nation's Children's Fund, Nepal Country Office, UN House Pulchowk,

Lalitpur, Nepal

Full list of author information is available at the end of the article
}

1.47 million neonatal deaths, which were directly due to other causes (e.g., infection) [2].

In 2012, an estimated 15 million babies (11.3\% of live births) worldwide were born preterm, about 13 million of these infants survived beyond the first month of life [2-5]. A large proportion of these preterm births $(37.6 \%)$ occurred in South Asia, for a prevalence rate of $13.3 \%$ among all live births in the region [6].

A meta-analysis on the association between neonatal mortality and preterm birth in East Africa showed that babies born at $<34$ weeks gestation had a 58 -fold increased risk for neonatal death than babies who were born 
at term. Further, babies who were born between 34 and 36 weeks of gestation had 3.2 times higher risk for neonatal death than babies who were born at term [7].

An infant is considered small for gestational age (SGA) when they are born with a birth weight below the tenth percentile according to a particular gestational age and sex-specific reference [8]. SGA can occur among infants who grew healthily in utero, but were naturally small. Alternatively, SGA can occur among infants who suffered from intrauterine growth restriction, which can be caused by a number of factors including placental insufficiency, environmental exposures, nutritional factors, etc. [9].

In 2010, 32.4 million SGA infants were born worldwide, of which 2.8 million were also preterm [3]. Approximately 706,200 deaths were attributable to SGA, globally. More than half of SGA births occurred in South Asia, where the prevalence of SGA was $44.5 \%$ and the prevalence of preterm and SGA births was $2.9 \%$ in 2010 [6]. In 2012, more than $80 \%$ of neonatal deaths in Sub-Saharan Africa and South Asia were among small babies $(65 \%$ attributable to preterm birth and $19 \%$ to term-SGA)[6]. Two-thirds of neonatal deaths among SGA infants were of term, low birth weight babies [2].

Nepal, a low-income country in South Asia, has a neonatal mortality rate of 24 per thousand live births, with more than half of these deaths caused by preterm birthrelated complications [1]. In 2010, $14 \%$ of babies were born preterm and $39.3 \%$ were SGA at birth in Nepal [6]. By determining the level of risk for neonatal death in babies born prematurely and/or SGA in a hospital setting in Nepal, we can provide evidence of the need for improved quality of care for these infants through investments in evidence-based, low-cost interventions to increase survival. Therefore, we conducted this assessment to identify the association between neonatal mortality and preterm birth and/or SGA among babies born in a tertiary hospital of Nepal.

\section{Methods \\ Design}

We conducted a case-control study, nested within a larger hospital-based prospective cohort study aiming to evaluate the impact of a simplified neonatal resuscitation protocol on perinatal outcomes. For the purpose of the larger study, a reference population was selected from the source population (all admissions to the hospital for delivery) to assess the change in perinatal outcomes over a specified period of time. The reference population of the larger study was a randomly selected $20 \%$ of the women delivering in the hospital. This random selection was done using a lottery technique at the time of admission. For the purpose of this study, all live births from the reference population were selected to be in the referent population and all neonatal deaths occurring during the study period were included in the case population. Any neonatal death that occurred within the referent population was removed from that population and reclassified into the case population. The sample size for the study was based on the larger study and calculated to detect a $20 \%$ reduction in perinatal mortality, with a statistical power of $80 \%$ and level of significance at $5 \%$ [10]. This case-control study was conducted from July 1 , 2012 to September 30, 2013.

\section{Setting}

We conducted this study in a tertiary hospital, Paropakar Maternity and Women's Hospital, located in Kathmandu, Nepal. This government-funded hospital has 415 beds, with 407 staff equipped to provide comprehensive obstetric and gynecological services. The hospital has three delivery units, as well as a Kangaroo Mother Care unit, special newborn care unit and neonatal intensive care unit for the care of small and sick babies born there. Around 22,000 deliveries take place annually, with an incidence of preterm birth and low birth weight at 9 and $11 \%$, respectively in 2012, and a neonatal mortality rate of 9 per thousand live births [11].

As part of the larger study evaluating the impact of neonatal resuscitation protocol implementation, the study received approval from the Hospital's Institutional Review Committee, the Nepal Health Research Council (Reg. No. 37/2012) and the Ethical Review Board of Uppsala University (dnr 2012/267). The study was registered as clinical trial, ISRCTN 97846009 [10]. Written consent was taken from all women who participated in the study.

\section{Participants}

All women with a neonatal death occurring during their stay in the hospital, throughout the study period were included as cases. Randomly selected women in the reference population (from original study), with live births were included in the referent population and were followed upto 28 days of birth. Any antepartum or intrapartum stillbirths occurring in the referent population were excluded from the study. Any neonatal death occurring in the referent population was excluded from this group, re-categorized, and included in the case population.

\section{Data collection}

A surveillance system was set up to collect sociodemographic, obstetric and postpartum information from the women in the case and referent populations at the admission, delivery and postnatal units. A surveillance team member at the admission unit collected information from the women who were admitted to the hospital for delivery. The team randomly selected $20 \%$ of women admitted to the hospital using a lottery 
technique. The surveillance team members at the delivery and postnatal units followed the referent women until discharge and followed up on the birth outcome through telephone interview conducted 28 days after delivery. The surveillance team at the delivery and postnatal units also collected information on the case population, i.e. all neonatal deaths that occurred in the hospital. Information about the case and referent populations was taken from the women's individual client journals, including demographic characteristics, obstetric history, intrapartum clinical progress and outcomes, and neonatal information. For certain socio-economic information, short interviews were completed with the women from the case and referent populations.

\section{Variables}

Neonatal mortality: Death of an infant from the time of birth until 28 days.

Preterm birth: Babies born before 37 completed weeks of gestation, estimated by the date of the mother's last menstrual period or based on clinical examination of the newborn.

Term birth: Babies who were born at, or after, 37 completed weeks of gestation, estimated by the mother's last menstrual period or based on clinical examination of the newborn.

Small for gestational age (SGA): Babies whose birth weight was less than the 10th percentile according to the appropriate gestational age and sex-specific reference population standards [8].

Appropriate for gestational age (AGA): Babies whose birth weight was greater than or equal to the 10th percentile according to the appropriate gestational age and sex-specific reference population standards [8].

Low Birth Weight (LBW): Babies who weighed less than 2500 grams at the time of birth.

Wealth quintile: The wealth index is a measure of socioeconomic position, used in nationally representative health surveys (Demographic Health Surveys) to compare the socio-economic inequalities $[12,13]$. During the interviews with mothers, data was collected on ownership of durable assets (e.g. car, refigerator, bicycle, radio, television), housing characteristics (e.g. number of rooms, dwelling floor and roof materials, toilet facilities) and access to services (e.g. electricity supply, drinking water source). Using the scores from the first principal component analysis, a wealth index (asset index) was contructed. Based on the value of the index, individuals were sorted and established to create cut-off values for percentiles within the population. These quintiles were then ranked from bottom to top as poorest, poorer, middle, richer and richest [14].

Ethnicity: The group within the social hierarchical system of Nepal to which the women's family belongs [15].
Parity: Number of times a woman has given birth after the age of viability, i.e. 22 weeks, including both live and still births [16].

Antenatal care attendance: The number of antenatal care visits that a woman went to in order to receive antenatal care from a skilled health worker.

Obstetric complication during pregnancy: Any complication that a woman had during the pregnancy period [17], including the following:

Antepartum hemorrhage: Excessive vaginal bleeding occurring before the onset of labor.

Hypertensive disorder during pregnancy: Classified by maternal diastolic blood pressure greater than or equal to $90 \mathrm{mmHg}$ in two different recordings, at least $4 \mathrm{~h}$ apart.

Multiple pregnancies: When a woman was pregnant with more than one fetus.

Medical disorder: When a woman had any of the following: diabetes mellitus, severe anemia $(\mathrm{Hb}<7 \mathrm{gm} / \mathrm{L})$, epilepsy, or other serious medical condition during pregnancy.

Obstetric complication during delivery: Any complication that a woman had during the intrapartum period [17], including the following:

Hypertensive disorder: Classified by maternal diastolic blood pressure greater than or equal to $90 \mathrm{mmHg}$ in two separate recordings.

Mal-presentation: Presentation of the fetus in any position besides vertex, i.e. with the top of the head appearing first.

Prolonged labor: When cervical dilation did not move beyond $4 \mathrm{~cm}$ after $8 \mathrm{~h}$ of regular contractions, or if cervical dilation was to the right of the alert line on the partogram.

Prolapsed cord: Characterized by the presence of the umbilical cord in the birth canal below the fetal presenting part, or at the vagina following the rupture of membranes.

\section{Data analysis}

The demographic, social and obstetric characteristics of the case and referent populations were compared using a Pearson's chi-square test, Wilcoxon rank-sum $t$-test or Fisher's exact test to assess whether there was a difference $(p<0.05)$ between the two groups.

For comparison of the demographic, social and obstetric characteristics of the case and referent populations, categorical variables were created. Maternal age was categorized into 5-year intervals including $\leq 20,21-25$, $26-30$, and $>30$ years; maternal education was categorized as less than 6 years of education or greater than or equal to 6 years of education; ethnicity was classified into six groups as Brahmin/Chhetri (hill and terai), relatively advantaged Janajatis (Newar, Gurung, Thakali), disadvantaged Janajatis, Dalit (hill and terai), non-Dalit terai, and Muslims; wealth was classified into five population quintiles: poorest, poorer, middle, richer and 
richest; parity was classified into three groups: primiparous, multiparous (1-2) and multiparous (3 or more); antenatal care attendance was classified as having attended any antenatal care or none; obstetric complications during pregnancy were classified as having any or having none; obstetric complications during the intrapartum period were also classified as having any versus none; the number of babies was categorized as multiple pregnancy or not (i.e., singleton); the sex of the baby as male or female; mode of delivery as vaginal, instrumental or cesarean section; gestational age at birth was classified as term versus preterm; and size according to gestational age was classified as SGA or AGA. We also created a binary variable grouping babies who were born both preterm and SGA versus those who were neither.

Univariate logistic regression analysis was done to test the association between neonatal death and demographic, social and obstetric characteristics of the women and babies that showed differences $(p<0.01)$ between the case and referent populations. Three different multivariable models were created to assess the level of association of neonatal mortality with preterm and/or SGA after adjusting for maternal age, maternal educational status, antenatal care attendance, wealth status, complication during the intrapartum period, mode of delivery, parity and multiple pregnancy. The first multivariable model assessed the level of association between neonatal mortality and preterm birth compared to term; the second multivariable model assessed the level of association between neonatal mortality and being born SGA compared to babies born AGA; and, the third model assessed the level of association between neonatal mortality and being born both preterm and SGA compared to being born only preterm, or only SGA, or neither.

We used the multiple imputation method to deal with data missing at random from the case or referent populations for the demographic, social, and/or obstetric variables [18].

\section{Results}

During the study period, there were 25,108 women who delivered in the hospital and a total of 299 neonatal deaths, giving a neonatal mortality rate of 11.9 per thousand live births. A total of 4,413 referent babies were alive at the time of their discharge from the hospital and thus included in the referent population (Fig. 1).

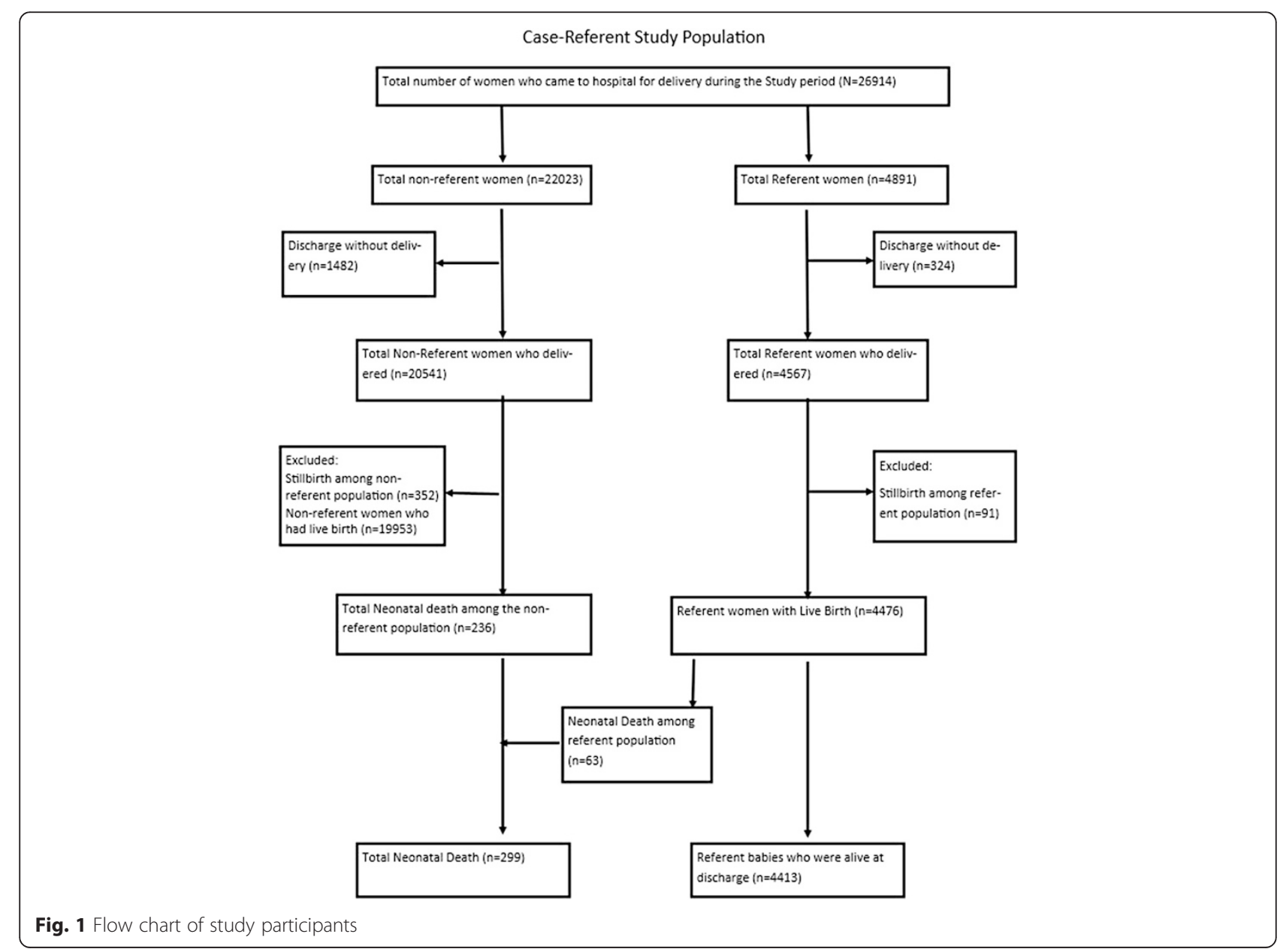


Table 1 Demographic, social and obstetric characteristics of live births (referent population) and neonatal deaths (case population)

\begin{tabular}{|c|c|c|c|}
\hline Characteristics & Live births $(n=4413$ ) & Neonatal deaths $(n=299)$ & $p$-value ${ }^{*}$ \\
\hline \multicolumn{4}{|l|}{ Maternal age (years) } \\
\hline Mean \pm standard deviation & $23.7 \pm 4.4$ & $24.3 \pm 5.6$ & $p=0.71$ \\
\hline Median (interquartile range) & $23(20-26)$ & $23(20-28)$ & $p=0.63$ \\
\hline Intervals: & n (\%) & n (\%) & \\
\hline$\leq 20$ & $1204(27.3)$ & $85(28.4)$ & \\
\hline $21-25$ & $1931(43.8)$ & $120(40.1)$ & \\
\hline $26-30$ & $961(21.8)$ & $49(16.4)$ & \\
\hline$>30$ & $317(7.2)$ & $45(15.1)$ & $p<0.001$ \\
\hline \multicolumn{4}{|l|}{ Ethnicity } \\
\hline Brahmin/Chhetri (Hill and Terai) & $1710(38.7)$ & $110(36.8)$ & \\
\hline Relatively advantaged Janajatis & $804(18.2)$ & $52(17.4)$ & \\
\hline Disadvantaged Janajatis & $1274(28.9)$ & $84(28.1)$ & \\
\hline Non-Dalit Terai & $363(8.2)$ & $31(10.4)$ & \\
\hline Dalit (Hill and Terai) & $230(5.2)$ & $16(5.4)$ & \\
\hline Muslim & $32(0.7)$ & $6(2.0)$ & $p=0.174$ \\
\hline \multicolumn{4}{|l|}{ Maternal education } \\
\hline Six years of education or less & $70(23.4)$ & $1448(32.8)$ & \\
\hline More than 6 years of education & $229(76.6)$ & $2965(67.2)$ & $p=0.001$ \\
\hline \multicolumn{4}{|l|}{ Wealth index (quintiles) ${ }^{a}$} \\
\hline Poorest & $768(18.7)$ & $67(39.2)$ & \\
\hline Poorer & $802(19.6)$ & $26(15.2)$ & \\
\hline Middle & $857(20.9)$ & $29(17.0)$ & \\
\hline Richer & $830(20.2)$ & $29(17.0)$ & \\
\hline Richest & $843(20.6)$ & $20(11.7)$ & $p<0.001$ \\
\hline \multicolumn{4}{|l|}{ Parity } \\
\hline Primiparous & $2385(54.0)$ & $146(48.8)$ & \\
\hline Multiparous (1-2) & $1845(41.8)$ & $118(39.5)$ & \\
\hline Multiparous ( $\geq 3$ ) & $183(4.1)$ & $35(11.7)$ & $p<0.001$ \\
\hline \multicolumn{4}{|l|}{ Antenatal care attendance } \\
\hline Yes & $3857(87.4)$ & $174(58.2)$ & \\
\hline No & $556(12.6)$ & $125(41.8)$ & $p<0.001$ \\
\hline \multicolumn{4}{|c|}{ Obstetric complication(s) in antepartum period } \\
\hline No & $3267(74.0)$ & $233(77.9)$ & \\
\hline Yes & $1146(26.0)$ & $66(22.1)$ & $p<0.151$ \\
\hline \multicolumn{4}{|l|}{ Multiple pregnancy } \\
\hline No & $4378(99.2)$ & $283(94.6)$ & \\
\hline Yes & $35(0.8)$ & $16(5.4)$ & $p<0.001$ \\
\hline \multicolumn{4}{|c|}{ Obstetric complication(s) in intrapartum period } \\
\hline No & $3923(88.9)$ & $194(64.9)$ & \\
\hline Yes & $490(11.1)$ & $105(35.1)$ & $p<0.001$ \\
\hline \multicolumn{4}{|l|}{ Mode of delivery } \\
\hline Vaginal & 3418 (77.5) & $195(65.2)$ & \\
\hline Cesarean section & 995 (22.5) & $104(34.8)$ & $p<0.001$ \\
\hline
\end{tabular}


Table 1 Demographic, social and obstetric characteristics of live births (referent population) and neonatal deaths (case population) (Continued)

\begin{tabular}{|c|c|c|c|}
\hline \multicolumn{4}{|l|}{ Sex } \\
\hline Male & $2333(52.9)$ & $176(58.9)$ & \\
\hline Female & $2080(47.1)$ & $123(41.1)$ & $p=0.074$ \\
\hline \multicolumn{4}{|l|}{ Preterm (<37 weeks) } \\
\hline No & $4079(92.4)$ & $116(38.8)$ & \\
\hline Yes & $334(7.6)$ & $183(61.2)$ & $p<0.001$ \\
\hline \multicolumn{4}{|l|}{ Birth weight (grams) } \\
\hline Mean \pm standard deviation & $2934.2 \pm 473.4$ & $1853.7 \pm 796.8$ & $p<0.001$ \\
\hline \multirow[t]{2}{*}{ Median (interquartile range) } & $3000(2600-3250)$ & $1650(1200-2400)$ & $p<0.001$ \\
\hline & n (\%) & n (\%) & \\
\hline \multicolumn{4}{|c|}{ Small for gestational age (<10th percentile) } \\
\hline No & $2772(62.8)$ & $156(52.2)$ & \\
\hline Yes & $1641(37.2)$ & $143(47.8)$ & $p<0.001$ \\
\hline \multicolumn{4}{|c|}{ Preterm and Small for gestational age } \\
\hline No & 4349 (98.5) & $239(79.9)$ & \\
\hline Yes & $64(1.5)$ & $60(20.1)$ & $p<0.001$ \\
\hline
\end{tabular}

${ }^{*} p$-value was determined by Pearson's chi-square test, Wilcoxon rank-sum t-test or Fisher's exact test

${ }^{a}$ There are some missing values for the wealth index variable: 313 missing among the live birth group (referent population) and 128 missing in the neonatal death group (case population)

During this time, the hospital had an incidence rate of preterm birth and SGA of 8.1 and $37.5 \%$, respectively.

The mean maternal ages of the case and referent populations were 24.3 and 23.7 years, respectively, which was significantly different between the two groups $(p<0.001)$. Women with less education, who were from the poorest family, multiparous, or who had no antenatal care had more neonatal deaths $(p<0.001)$. Similarly, there were more neonatal deaths among women with obstetric complications during the intrapartum period than those women without $(p<0.001)$. There were also more neonatal deaths among babies who were born prematurely, with a low birth weight or SGA than babies who were born at term, with a normal birth weight or AGA $(p<0.001)$ (Table 1$)$.

Univariate logistic regression analysis assessing the likelihood for neonatal mortality based on maternal demographic characteristics showed that older woman, those with less education (6 years or less) or from the poorest families had an increased risk for neonatal mortality. For analyses based on obstetric characteristics, those women who were multiparous, who had no antenatal care from a skilled provider, had obstetric complications during the intrapartum period, who had a multiple pregnancy or Cesarean section also had a higher risk of neonatal mortality. The level of risk for neonatal mortality increased by $50 \%$ if the baby was SGA, for preterm babies the risk increased 17 -fold, and there was a 19-fold increased risk if the baby was both preterm and SGA (Table 2).
In the multivariable logistic regression models, the following characteristics were adjusted for, as they were different between the case and referent populations: maternal age, maternal educational status, wealth status, parity, antenatal care attendance, multiple pregnancy, obstetric complication during the intrapartum period, and mode of delivery. After adjusting for these potential confounders, the likelihood for neonatal death was 12 times higher for babies who were preterm than those who were born at term, $40 \%$ higher for SGA compared to AGA infants and 16 times higher for babies who were both preterm and SGA (Table 3).

\section{Discussion}

Our study showed that the risk of neonatal mortality was highest among babies born both preterm and SGA, followed by babies born preterm, and then by SGA babies in Nepal. Babies who are born prematurely or SGA have an increased risk for hypothermia, infection, respiratory distress syndrome (RDS), intracranial hemorrhage, necrotizing enterocolitis, retinopathy of prematurity, neurodevelopmental impairment and mortality $[9,19,20]$. These complications could potentially be prevented, or minimized, with interventions like Kangaroo Mother Care and extra-support for feeding, case management of babies with signs of infection, safe oxygen management and supportive care for RDS, hospital care of babies with RDS, use of continuous positive airway pressure (CPAP) and surfactant, or intensive neonatal care [21-24]. 
Table 2 Logistic regression analyses for likelihood of neonatal death based on selected maternal and infant characteristics

\begin{tabular}{|c|c|c|}
\hline & $\begin{array}{l}\text { Crude Odds } \\
\text { Ratio }(\mathrm{COR})^{\mathrm{a}}\end{array}$ & $95 \% \mathrm{Cl}$ \\
\hline \multicolumn{3}{|l|}{ Maternal age (years) } \\
\hline Maternal age & Ref & \\
\hline Increase in age & 1.02 & $1.0-1.1$ \\
\hline \multicolumn{3}{|l|}{ Maternal education } \\
\hline More than 6 years of education & Ref & \\
\hline Education of less than 6 years & 1.6 & $1.2-2.1$ \\
\hline \multicolumn{3}{|l|}{ Wealth status $^{\mathrm{b}}$} \\
\hline Non-poor & Ref & \\
\hline Poor & 1.4 & $1.0-1.8$ \\
\hline \multicolumn{3}{|l|}{ Parity } \\
\hline Primi-parous & Ref & \\
\hline Multi-parous & 1.4 & $1.2-1.5$ \\
\hline \multicolumn{3}{|l|}{ Antenatal care attendance } \\
\hline Yes & Ref & \\
\hline No & 5.0 & $3.9-6.4$ \\
\hline \multicolumn{3}{|l|}{ Multiple pregnancy } \\
\hline No & Ref & \\
\hline Yes & 7.1 & $3.9-12.9$ \\
\hline \multicolumn{3}{|c|}{$\begin{array}{l}\text { Obstetric complication during intrapartum } \\
\text { period }\end{array}$} \\
\hline No & Ref & \\
\hline Yes & 4.3 & $3.4-5.6$ \\
\hline \multicolumn{3}{|l|}{ Mode of delivery } \\
\hline Vaginal delivery & Ref & \\
\hline Cesarean section & 1.8 & $1.4-2.4$ \\
\hline \multicolumn{3}{|l|}{ Preterm (<37 weeks of gestation) } \\
\hline No & Ref & \\
\hline Yes & 17.1 & $11.7-24.8$ \\
\hline \multicolumn{3}{|c|}{ Small for gestational age ( $<10$ th percentile) } \\
\hline No & Ref & \\
\hline Yes & 1.5 & $1.2-2.0$ \\
\hline \multicolumn{3}{|l|}{ Preterm and Small for Gestational Age } \\
\hline No & Ref & \\
\hline Yes & 19.3 & $14.9-25.0$ \\
\hline \multicolumn{3}{|c|}{$\begin{array}{l}\text { anivariate logistic regression analysis done to test the association between } \\
\text { neonatal death and characteristics of the women and babies that showed } \\
\text { differences }(p<0.01) \text { among the case and referent populations } \\
\text { bThe women from the poorest wealth quintile were categorized as poor and } \\
\text { the remaining women in the poorer to richest quintiles were categorized } \\
\text { as non-poor }\end{array}$} \\
\hline
\end{tabular}

There were some potential limitations within this study. First, there may have been some bias within this study, such as the failure of the health worker to correctly assess the medical condition during clinical examination at admission or during delivery. Second, the
Table 3 Multivariable regression analysis for risk of neonatal death for babies born preterm, small weight for gestational age (SGA) or both

\begin{tabular}{|c|c|c|}
\hline & Adjusted Odds Ratio ${ }^{a}$ & $95 \% \mathrm{Cl}$ \\
\hline \multicolumn{3}{|c|}{ Preterm ( $<37$ weeks of gestation) } \\
\hline No & Ref & \\
\hline Yes & 12.4 & $8.1-18.9$ \\
\hline \multicolumn{3}{|c|}{ SGA (<10th percentile) } \\
\hline No & Ref & \\
\hline Yes & 1.4 & $1.1-1.8$ \\
\hline \multicolumn{3}{|c|}{ Preterm and SGA } \\
\hline No & Ref & \\
\hline Yes & 16.2 & $12.3-21.3$ \\
\hline
\end{tabular}

${ }^{a}$ Multivariable logistic regression analysis for the likelihood of neonatal death, adjusted for maternal age, maternal educational status, antenatal care attendance, wealth status, complication(s) during the intrapartum period, mode of delivery, parity, and multiple pregnancy

gestational age estimate we used was based on the last maternal menstrual period, so there may have been some recall bias from the women. Third, the weight for gestational age references used were based on a US national population, which are likely different from what the Nepal national population references would be. Fourth, the telephone follow ups were only done for referent population, so the neonatal death occurring in the non-referent population $(80 \%)$ after discharge were missed, which could potentially result in underreporting of neonatal mortality due to prematurity or small for gestational age. Finally, this is a hospital-based study, and the population coming to this tertiary referral hospital may not be representative of the larger population in Nepal. However, the objective of this paper was to assess the level of risk for neonatal death among infants who were either preterm and/or SGA in a tertiary hospital setting, so that in the future, the quality of care provided for these infants can be improved.

A pooled analysis conducted using data from low- and middle-income countries to determine the risk for neonatal mortality among preterm and SGA infants showed that babies who were preterm had a 6.8 times higher risk for death than babies born term; SGA babies had a 1.83 times increased risk for neonatal death compared to AGA; and babies who were both had a 15-fold increased risk for neonatal mortality [25]. Our study showed the level of risk for neonatal mortality among these same groups to be higher than the pooled analysis conducted in other low- and middle-income countries.

In Nepal, more than half of total deliveries take place at health institutions, and care seeking for sick and small babies at referral hospitals has increased in the last 10 years $[26,27]$. Together with this increased use of referral hospitals for delivery and postnatal care, the 
findings of increased likelihood for neonatal death among high-risk infants from our study, done in a referral hospital setting, indicate that interventions for reducing preterm- and/or SGA-complication related mortality have not yet been extensively implemented. As a country, Nepal has committed to reduce the neonatal mortality rate to 10 (or less) per thousand live births by 2035 at the World Health Assembly 2014 as part of the Every Newborn Action Plan [28]. Based on our estimates, the current proportion of neonatal deaths due to preterm- and/or SGA-related complications has to be reduced by half, in order to meet this goal.

\section{Conclusion}

To our knowledge, this is the first study done in Nepal with the aim to determine the level of risk for neonatal death among babies who were born prematurely and/or SGA in a tertiary health facility. The increased risk for neonatal death among preterm and/or SGA infants together with the increased trend for institutional delivery, reveals the need for furthered investment in the scale up of evidence-based interventions such as Kangaroo Mother Care or antenatal corticosteroids, as well as the improvement of special newborn care and neonatal intensive care units. Periodic analysis on the level of risk for neonatal mortality among preterm and SGA babies in health facilities can provide a reflection of the efficiency of the implementation of such interventions to reduce preterm- and SGA-related deaths.

\section{Competing interests}

The authors declare that they have no competing interest.

\section{Authors' contributions}

AK, MM, JW, and UE conceptualized and designed the study. AK was the principal applicant for funding. AK was responsible for the data collection and data management. AK, VN and MM were responsible for the data analysis and drafting of the manuscript. JW, UE and RC reviewed and provided input on the manuscript draft. All authors read and approved the final manuscript.

\section{Acknowledgements \\ The study group would like to thank Ravi Vitrakoti, Dr. Sheela Verma, Dr. Gehanath Baral and Dr. Dhan Raj Aryal from Paropakar Maternity and Women's hospital who reviewed and provided input for the design and data collection methods of this study. The Laerdal Foundation supported this study for Acute Medicine (Stavanger, Norway) as part of the larger study to evaluate the impact of the implementation of a simplified neonatal resuscitation protocol on birth outcomes and health worker performance. The Laerdal foundation for Acute Medicine had no influence on conceptualization or design of this study. MM, JW, UE and VN were supported through a university grant from Uppsala University (Uppsala, Sweden). The group is very grateful to the mothers who participated in the study.}

\section{Author details}

${ }^{1}$ International Maternal and Child Health, Department of Women's and Children's Health, Uppsala University, Uppsala, Sweden. ${ }^{2}$ United Nation's Children's Fund, Nepal Country Office, UN House Pulchowk, Lalitpur, Nepal. ${ }^{3}$ Latter Day Saints Charities, Salt Lake City, Utah, USA.

Received: 12 May 2015 Accepted: 4 September 2015

Published online: 10 September 2015

\section{References}

1. UNICEF WHO, The World Bank, United Nations. Levels and trends in child mortality; report 2013. In: UNICEF W, editor. The World Bank, United Nations. NewYork, USA: UNICEF; 2013.

2. Lawn JE, Blencowe H, Oza S, You D, Lee AC, Waiswa P, et al. Lancet every newborn study group: every newborn: progress, priorities, and potential beyond survival. Lancet. 2014;384(9938):189-205.

3. Blencowe $H$, Cousens S, Oestergaard MZ, Chou D, Moller AB, Narwal R, et al. National, regional, and worldwide estimates of preterm birth rates in the year 2010 with time trends since 1990 for selected countries: a systematic analysis and implications. Lancet. 2012;379(9832):2162-72.

4. Blencowe $H$, Cousens S, Chou D, Oestergaard M, Say L, Moller AB, et al. Born too soon: the global epidemiology of 15 million preterm births. Reprod Health. 2013;10 Suppl 1:S2.

5. Kinney MV, Lawn JE, Howson CP, Belizan J. 15 Million preterm births annually: what has changed this year? Reprod Health. 2012;9:28

6. Lee AC, Katz J, Blencowe H, Cousens S, Kozuki N, Vogel JP, et al. National and regional estimates of term and preterm babies born small for gestational age in 138 low-income and middle-income countries in 2010. Lancet Glob Health. 2013;1(1):e26-36.

7. Marchant T, Willey B, Katz J, Clarke S, Kariuki S, ter Kuile F, et al. Neonatal mortality risk associated with preterm birth in East Africa, adjusted by weight for gestational age: individual participant level meta-analysis. PLoS Med. 2012;9(8):e1001292.

8. Alexander GR, Himes JH, Kaufman RB, Mor J, Kogan M. A United States national reference for fetal growth. Obstet Gynecol. 1996;87(2):163-8.

9. Lawn JE, Davidge R, Paul VK, von Xylander S, de Graft Johnson J, Costello A, et al. Born too soon: care for the preterm baby. Reprod Health. 2013;10 Suppl 1:S5.

10. Ashish KC, Malqvist M, Wrammert J, Verma S, Aryal DR, Clark R, et al. Implementing a simplified neonatal resuscitation protocol-helping babies breathe at birth (HBB) - at a tertiary level hospital in Nepal for an increased perinatal survival. BMC Pediatr. 2012;12:159.

11. Ministry of Health and Population, GoN: Annual Report "Smarika" 2068-69. Kathmandu, Nepal: 2012

12. Howe LD, Hargreaves JR, Gabrysch S, Huttly SR. Is the wealth index a proxy for consumption expenditure? A systematic review. J Epidemiol Community Health. 2009;63(11):871-7.

13. Rutsein SO, Johnson K. The DHS wealth index- DHS Comparative Reports No. 6. ORC Macro: Calverton, Maryland; 2004.

14. Filmer D, Pritchett LH. Estimating wealth effects without expenditure data-or tears: an application to educational enrollments in states of India. Demography. 2001;38(1):115-32.

15. World Bank, DFID. Unequal Citizens: Gender, Caste and Ethnic Exclusion in Nepal -Summary Report. Kathmandu: World Bank, Department for International Development (DFID); 2006.

16. Opara El, Zaidi J. The interpretation and clinical application of the word 'parity': a survey. BJOG. 2007;114(10):1295-7.

17. World Health Organization. Managing complications in pregnancy and childbirth: a guide for midwives and doctors. In: Edited by WHO. World Health Organization: 20 Avenue Appia, 1211 Geneva 27, Switzerland; 2000.

18. Barnard J, Meng XL. Applications of multiple imputation in medical studies: from AIDS to NHANES. Stat Methods Med Res. 1999;8(1):17-36.

19. Blencowe H, Lee AC, Cousens S, Bahalim A, Narwal R, Zhong N, et al. Preterm birth-associated neurodevelopmental impairment estimates at regional and global levels for 2010. Pediatr Res. 2013;74 Suppl 1:17-34.

20. Blencowe H, Lawn JE, Vazquez T, Fielder A, Gilbert C. Preterm-associated visual impairment and estimates of retinopathy of prematurity at regional and global levels for 2010. Pediatr Res. 2013;74 Suppl 1:35-49.

21. Finer NN, Carlo WA, Walsh MC, Rich W, Gantz MG, Laptook AR, et al. Early CPAP versus surfactant in extremely preterm infants. N Engl J Med. 2010;362(21):1970-9.

22. Conde-Agudelo A, Diaz-Rossello JL. Kangaroo mother care to reduce morbidity and mortality in low birthweight infants. Cochrane Database Syst Rev. 2014:4:CD002771.

23. Vidyasagar D, Velaphi S, Bhat VB. Surfactant replacement therapy in developing countries. Neonatology. 2011;99(4):355-66.

24. Bhutta ZA, Das JK, Bahl R, Lawn JE, Salam RA, Paul VK, et al. Can available interventions end preventable deaths in mothers, newborn babies, and stillbirths, and at what cost? Lancet. 2014;384(9940):347-70.

25. Katz J, Lee AC, Kozuki N, Lawn JE, Cousens S, Blencowe $H$, et al. CHERG-Preterm Birth Working Group: Mortality risk in preterm and small-for- 
gestational-age infants in low-income and middle-income countries: a pooled country analysis. Lancet. 2013;382(9890):417-25.

26. UNICEF, GoN. Multiple indicator cluster survey (MICS). Nepal: UNICEF; 2015

27. Ministry of Health and Population, GoN. Responding to increased demand for institutional childbirths at referral hospitals in Nepal: situational analysis and emerging options 2013. Kathmandu: Family Health Division; 2013.

28. WHO, UNICEF. Every Newborn: an action plan to end preventable deaths. Geneva: World Health Organization; 2014.

Submit your next manuscript to BioMed Central and take full advantage of:

- Convenient online submission

- Thorough peer review

- No space constraints or color figure charges

- Immediate publication on acceptance

- Inclusion in PubMed, CAS, Scopus and Google Scholar

- Research which is freely available for redistribution 